Western University

Scholarship@Western

Brain and Mind Institute Researchers'

Publications

Brain and Mind Institute

$10-15-2016$

\title{
Usage of SWI (susceptibility weighted imaging) acquired at 7T for qualitative evaluation of temporal lobe epilepsy patients with histopathological and clinical correlation: An initial pilot study.
}

\author{
Benjamin Y M Kwan \\ Department of Medical Imaging, Schulich School of Medicine and Dentistry, Western University, 1151 \\ Richmond St. North, London, Ontario, N6A 5B7, Canada \\ Fateme Salehi \\ Department of Medical Imaging, Schulich School of Medicine and Dentistry, Western University, 1151 \\ Richmond St. North, London, Ontario, N6A 5B7, Canada \\ Pavlo Ohorodnyk \\ Department of Medical Imaging, Schulich School of Medicine and Dentistry, Western University, 1151 \\ Richmond St. North, London, Ontario, N6A 5B7, Canada \\ Donald H Lee \\ Department of Medical Imaging, Schulich School of Medicine and Dentistry, Western University, 1151 \\ Richmond St. North, London, Ontario, N6A 5B7, Canada \\ Jorge G Burneo \\ Epilepsy Program, Department of Clinical Neurological Sciences, Schulich School of Medicine and

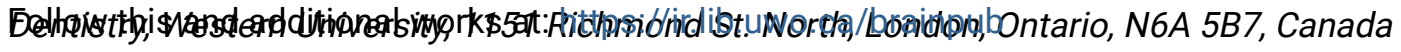 \\ Part of the Neurosciences Commons, and the Psychology Commons
}

See next page for aưưitional aưthors

Citation of this paper:

Kwan, Benjamin Y M; Salehi, Fateme; Ohorodnyk, Pavlo; Lee, Donald H; Burneo, Jorge G; Mirsattari, Seyed M; Steven, David; Hammond, Robert; Peters, Terry M; and Khan, Ali R, "Usage of SWI (susceptibility weighted imaging) acquired at 7T for qualitative evaluation of temporal lobe epilepsy patients with histopathological and clinical correlation: An initial pilot study." (2016). Brain and Mind Institute Researchers' Publications. 251.

https://ir.lib.uwo.ca/brainpub/251 


\section{Authors}

Benjamin Y M Kwan, Fateme Salehi, Pavlo Ohorodnyk, Donald H Lee, Jorge G Burneo, Seyed M Mirsattari, David Steven, Robert Hammond, Terry M Peters, and Ali R Khan 


\title{
Usage of SWI (susceptibility weighted imaging) acquired at $7 \mathrm{~T}$ for qualitative evaluation of temporal lobe epilepsy patients with histopathological and clinical correlation: An initial pilot study
}

\author{
Benjamin Y.M. Kwan ${ }^{\text {b }}$, Fateme Salehi ${ }^{\text {b }}$, Pavlo Ohorodnyk ${ }^{\text {b }}$, Donald H. Lee ${ }^{\text {b }}$, Jorge G. Burneo ${ }^{\mathrm{d}}$, \\ Seyed M. Mirsattari ${ }^{\mathrm{d}}$, David Steven ${ }^{\mathrm{d}}$, Robert Hammond ${ }^{\mathrm{e}}$, Terry M. Peters ${ }^{\mathrm{a}, \mathrm{b}, \mathrm{c}}$, Ali R. Khan ${ }^{\mathrm{a}, \mathrm{b}, \mathrm{c}, *}$ \\ a Imaging Research Laboratories, Robarts Research Institute, Western University, 1151 Richmond St. North, London, Ontario, N6A 5B7, Canada \\ ${ }^{\mathrm{b}}$ Department of Medical Imaging, Schulich School of Medicine and Dentistry, Western University, 1151 Richmond St. North, London, Ontario, N6A 5B7, Canada \\ c Department of Medical Biophysics, Schulich School of Medicine and Dentistry, Western University, 1151 Richmond St. North, London, Ontario, N6A 5B7, Canada

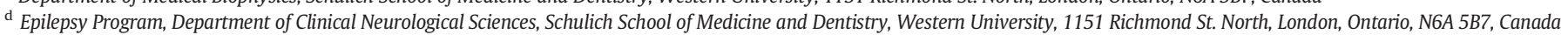 \\ e Department of Pathology and Laboratory Medicine, Schulich School of Medicine and Dentistry, Western University, 1151 Richmond St. North, London, Ontario, N6A 5B7, Canada
}

\section{A R T I C L E I N F O}

\section{Article history:}

Received 10 May 2016

Received in revised form 6 July 2016

Accepted 29 July 2016

Available online 2 August 2016

\section{Keywords:}

7 T MRI

Temporal lobe epilepsy

Histopathology

Neuroradiology

\begin{abstract}
A B S T R A C T
Objectives: Ultra high field MRI at $7 \mathrm{~T}$ is able to provide much improved spatial and contrast resolution which may aid in the diagnosis of hippocampal abnormalities. This paper presents a preliminary experience on qualitative evaluation of $7 \mathrm{~T}$ MRI in temporal lobe epilepsy patients with a focus on comparison to histopathology. Methods: 7 T ultra high field MRI data, using T1-weighted, T2*-weighted and susceptibility-weighted images (SWI), were acquired for 13 patients with drug resistant temporal lobe epilepsy (TLE) during evaluation for potential epilepsy surgery. Qualitative evaluation of the imaging data for scan quality and presence of hippocampal and temporal lobe abnormalities were scored while blinded to the clinical data. Correlation of imaging findings with the clinical data was performed. Blinded evaluation of $1.5 \mathrm{~T}$ scans was also performed.

Results: On the 7 T MRI findings, eight out of 13 cases demonstrated concordance with the clinically suspected TLE. Among these concordant cases, three exhibited supportive abnormal 7 T MRI findings which were not detected by the clinical $1.5 \mathrm{~T}$ MRI. Of the ten cases that progressed to epilepsy surgery, seven showed concordance between $7 \mathrm{~T}$ MRI findings and histopathology; of these, four cases had hippocampal sclerosis. SWI had the highest concordance with the clinical and histopathological findings. Similar clinical and histopathological concordance was found with $1.5 \mathrm{~T}$ MRI.

Conclusions: There was moderate and high concordance between the $7 \mathrm{~T}$ imaging findings with the clinical data and histopathology respectively.
\end{abstract}

(c) 2016 Elsevier B.V. All rights reserved.

\section{Introduction}

Epilepsy is the leading neurological disorder in terms of years of life lost to disease, with more than one-third of the patients being refractory to pharmacotherapy [1]. Temporal lobe epilepsy (TLE), and in particular hippocampal sclerosis (HS), is a common form of medically refractory epilepsy [2]. Surgical intervention is the standard of care in many of these patients. In those with TLE, a randomized control trial by Wiebe et al. showed that surgery results in seizure-free status in $58 \%$, compared to $8 \%$ with medical treatment [3]. In addition, surgical outcomes in patients with underlying lesions are more favourable [4,5]. In 20$30 \%$ of patients with refractory epilepsy, no structural abnormalities

\footnotetext{
* Corresponding author at: Robarts Research Institute, Departments of Medical Biophysics \& Medical Imaging, Western University, 1151 Richmond St N, London, ON N6A 5B7, Canada.

E-mail address: alik@robarts.ca (A.R. Khan).
}

are identified on MRI, and patients with no discernible MRI lesions are more likely to have their surgery delayed than those with perceptible lesions on MRI [2]. In the absence of detectable structural lesions on MRI, patients often undergo invasive EEG recordings including insertion of subdural electrodes, which are associated with a risk of complications, prolonged hospital stay, and higher costs [6,7]. Recent studies have shown that $3 \mathrm{~T}$ MRI (as compared to $1.5 \mathrm{~T}$ MRI) improved identification of lesional abnormalities in patients previously classified as 'nonlesional' in $5 \%$ of cases and changed the diagnosis or prognosis of the underlying abnormality in $26 \%$ of the studied patients [2].

Evaluation for the presence of hippocampal abnormalities at $1.5 \mathrm{~T}$ and even $3 \mathrm{~T}$ field strengths is limited by spatial resolution and insufficient contrast (Fig. 1) [8]. Features of HS on MR imaging include the presence of atrophy, disruption of internal architecture and hyperintense signal on T2-weighted MR images [9]. A normal-appearing hippocampus occurs in up to $40 \%$ of the cases based on post-surgical histopathological study of resected temporal lobes [10]. Recent studies 


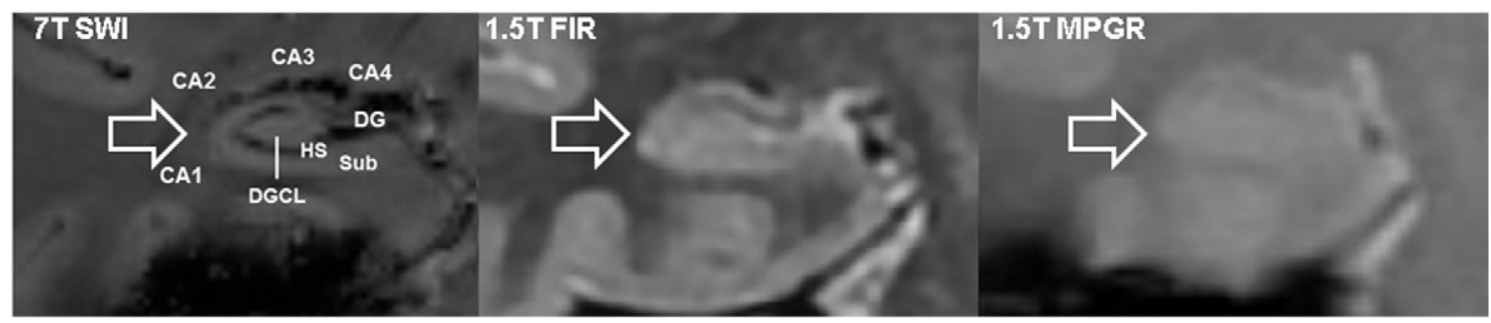

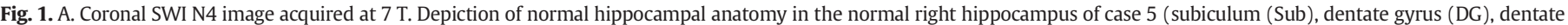

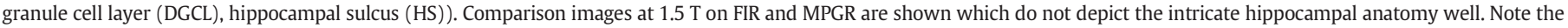
signal drop out at the inferior temporal lobe on $7 \mathrm{~T}$ which is a common artifact seen.

using 7 T MR imaging have shown its superior diagnostic benefits in various pathologies including multiple sclerosis, cerebrovascular diseases, aneurysms, cavernous malformations, polymicrogyria, and brain tumors [11,12]. T2*-weighted MR images acquired at $7 \mathrm{~T}$ have previously been shown to depict hippocampal subfield structures as small as $100 \mu \mathrm{m}$ [13]. However there are drawbacks to higher field strength imaging, including greater signal dropout in areas of local susceptibility differences (sinuses), and a higher level of inhomogeneities in the magnetic fields, which can cause distortions and signal loss artifacts.

The aim of the current study was to 1 ) determine the ability of $7 \mathrm{~T}$ MR imaging (and particularly susceptibility weighted images (SWI)) to detect hippocampal and mesial temporal lobe abnormalities in patients before temporal lobe resection and 2) evaluate concordance of $7 \mathrm{~T}$ MRI findings with post-operative histopathological results which can serve as a basis for validation of imaging findings.

\section{Methods}

Ethics approval was obtained through our institutional review board including participation of patients under 18 years of age. All human and animal studies have been approved by the appropriate ethics committee and have therefore been performed in accordance with the ethical standards laid down in the 1964 Declaration of Helsinki and its later amendments. Patients (male or female) with a history of drug-resistant TLE aged 16-65 years old were recruited for this study. Exclusion criteria included patients with severe coexisting or terminal systemic disease and those unsuitable for MRI evaluation. Thirteen patients undergoing evaluation for epilepsy were scanned using the 7 T MRI scanner. Each patient also underwent standard clinical 1.5 T MRI, prolonged video-EEG, and neuropsychological testing, as part of the clinical epilepsy evaluation at an academic tertiary care center. Seven patients also underwent invasive EEG electrode placement. Clinical data were collected for each patient, including age at surgery, gender, suspected epilepsy type, clinical 1.5 T MRI reports, EEG reports, subdural electrode recording reports, clinical follow-up notes post resection and corresponding histopathology reports if applicable.

Ultra high field data were acquired on a $7 \mathrm{~T}$ neuroimaging optimized MRI scanner (Agilent, Santa Clara, CA, USA/Siemens, Erlangen, Germany) using a 16-channel transmit-receive head coil array constructed in-house. The imaging sequences used for this study were a multiecho gradient-echo sequence with six echoes acquired with a $0.5 \mathrm{~mm}$ in-plane resolution $(\mathrm{TR}=40 \mathrm{~ms}, \mathrm{TE}=4.57 \mathrm{~ms}$, echo spacing $=$ $4.89 \mathrm{~ms}$, flip angle $=13$ degrees, $\mathrm{NEX}=1$, matrix $=256 \times 360,80$ slices, slice thickness $=1.5 \mathrm{~mm}$, in-plane resolution $0.5 \mathrm{~mm}$, FOV $=$ $128 \times 180 \times 120 \mathrm{~mm}$, acquisition time $=12 \mathrm{~min}$ ), with slices acquired perpendicular to the long axis of the hippocampus in a coronal oblique orientation, and a T1-weighted MPRAGE sequence (matrix = $256 \times 512 \times 172$, resolution $=0.58 \times 0.43 \times 1 \mathrm{~mm}$, acquisition time $=5: 42 \mathrm{~min}$ ). For the multi-echo image, the magnitude images for each of the six echoes were averaged to produce a $\mathrm{T}^{*}$-weighted image. SWI were generated from the multi-echo images using a frequency mask derived from the unwrapped and filtered phase images, using a process described in detail previously [14]. These volumes (T1,
T2*, SWI), three for each patient, had randomized identification numbers assigned and were converted to DICOM format for blinded review by a fellowship trained neuroradiologist with over 30 years of experience. Additionally, each volume was processed to remove shading artifact using a non-uniformity intensity normalization filter (N4) [15], which we refer to henceforth as N4, to assess the impact of viewing images with and without shading artifact removal.

Clinical MRI data was acquired on a 1.5 T MRI scanner (GE, USA). The imaging protocol for epilepsy imaging at this institution included T2 axial ( $2 \mathrm{~mm}$ contiguous slices, matrix $=256 \times 256 \mathrm{~mm}$, NEX $=1$, $\mathrm{TR}=12,666 \mathrm{~ms}, \mathrm{TE}=49 \mathrm{~ms}$, FOV $=22 \mathrm{~cm})$, FSEIR coronal (3.5/1, matrix $=288 \times 256$ Zipped to $512 \times 512, \mathrm{TR}=5116 \mathrm{~ms}, \mathrm{TE}=15 \mathrm{~ms}, \mathrm{TI}=$ $150 \mathrm{~ms}$, FOV $=22 \mathrm{~cm})$, gradient echo coronal $(5 / 1$, matrix $=$ $256 \times 192 \mathrm{~mm}, \mathrm{NEX}=2, \mathrm{FOV}=20 \mathrm{~cm}$ ), 3D FLAIR coronal (with $1.8 \mathrm{~mm}$ partitions, reconstructed at $0.9 \mathrm{~mm}$ intervals, $\mathrm{TR}=6000 \mathrm{~ms}$, $\mathrm{TE}=122 \mathrm{~ms}, \mathrm{TI}=1872 \mathrm{~ms}$, FOV $=24 \mathrm{~cm}$ ), 3D T1 axial ( with $2 \mathrm{~mm}$ partitions, reconstructed at $1 \mathrm{~mm}$ intervals, $\mathrm{FOV}=24 \mathrm{~cm}$, matrix $=$ $256 \times 256 \mathrm{~mm}$ ) and diffusion axial ((6 directions, B0, B1000), $5 \mathrm{~mm}$ contiguous, matrix $=128 \times 192, \mathrm{FOV}=24 \mathrm{~cm}, \mathrm{TR}=8100 \mathrm{~ms}, \mathrm{TE}=$ $88.8 \mathrm{~ms})$.

Each of the patient's 7 T MRI data was reviewed by the neuroradiologist using a qualitative grading scale while blinded to clinical information. Volumes were graded for scan quality and the presence of artifact. Qualitative grading of the mesial temporal lobe internal architecture (including note of signal abnormality and/or architectural abnormalities), mesial temporal lobe size and temporal neocortical architecture was performed (Table 1). The rest of the brain architecture was briefly evaluated for any gross abnormalities. The results of the neuroradiologist were converted into a single rating for each category by taking the most abnormal grade assigned for each category among the six volumes evaluated. Additionally, the grades of 'normal' or 'possibly normal' were merged into a single rating value of "normal" and the grades of "possibly abnormal' or 'definitely abnormal' were merged into a single rating value of "abnormal". A second reader (a senior radiology resident) also evaluated the $7 \mathrm{~T}$ MRI scans using the qualitative grading scale and assigned a single rating for each category while taking into account all the volumes. A Cohen's kappa was calculated between the two readers (GraphPad Software, La Jolla, CA, USA and StatsToDo, Australia).

The neuroradiologist also blindly evaluated the $1.5 \mathrm{~T}$ MRI data in a similar fashion.

Resected specimens were processed according to established protocol. Briefly, specimens were fixed in $10 \%$ buffered formalin. Fixed

Table 1

Qualitative rating scale.

\begin{tabular}{l} 
Merged rating of 'Normal' or 'Abnormal' (for each hemisphere) per category \\
\hline 1. Mesial temporal lobe internal architecture (including signal abnormalities) \\
2. Mesial temporal lobe size \\
3. Temporal neocortical architecture \\
4. Rest of brain architecture
\end{tabular}


material was serially blocked, sectioned and examined by routine histological stains (H\&E) and by immunoperoxidase for the expression of neuronal (NeuN) and glial (GFAP) antigens. Stained sections were examined by a neuropathologist and digitized.

Comparison of 7 T MRI findings (including location of abnormal findings) as interpreted by the neuroradiologist to the clinical data (including clinical history, multidisciplinary epilepsy group decisions, EEG reports and subdural electrode recording reports for seizure activity localization). Concordant correlation to histopathology was defined as any identified abnormal imaging finding with a corresponding abnormal histopathological finding on the ipsilateral side. Concordant HS was defined as abnormal mesial temporal lobe internal architecture and abnormal mesial temporal lobe size with corresponding MTS on histopathology. Clinical follow-up data was collected in regards to seizure free status if the patient underwent epilepsy surgery. Additionally, a similar comparison was made as detailed above but individually using each of the 6 volumes of data alone (T1, T2*, SWI, and their corresponding non-uniformity corrected data). Comparison of $1.5 \mathrm{~T}$ MRI findings was also performed in a similar fashion.

\section{Results}

Thirteen patients were included in the study ( 7 males, 6 females). Ten patients underwent epilepsy surgery ( 5 males, 5 females, average age of 33.4 years; median age 31.5 , range 18-50) and clinical followup was performed for an average of 25.6 months. Seven of ten cases undergoing epilepsy surgery had concordance between $1.5 \mathrm{~T}$ MRI findings with histopathology. Nine out of 13 cases had concordance of suspected clinical epilepsy syndrome with 1.5 T MRI findings.

Interrater agreement between the neuroradiologist and the second reader (for the 7 T MRI findings) calculated using the Cohen's Kappa was 0.6094 , SE 0.0971 (95\% CI 0.4191 to 0.7998 ) with the percentage of observed agreements at $86.5 \%$.

\subsection{Quality of scans and presence of artifacts}

Of the 78 volumes inspected (six volumes per patient), three were rendered non-diagnostic, due to the predominant artifacts of susceptibility and shading. Susceptibility artifacts were observed, particularly involving signal drop out affecting the inferior temporal lobe. Shading artifacts were partially mitigated on N4 correction, although ten of 37 corrected volumes were still deemed to be affected. Motion artifact affected three of the 13 cases.

\subsection{Concordance of clinical findings to 7 T MRI findings}

Eight of the 13 cases demonstrated concordance with the suspected clinical epilepsy syndrome as determined by the clinical management team (including data from neurological presentation, EEG and/or subdural electrode recordings) with the 7 T MRI findings. Among these concordant cases, three were deemed abnormal by the $7 \mathrm{~T}$ MRI findings that were supported by the clinical data, but not detected by the routine 1.5 T clinical MRI (cases 10,11 and 12) (Fig. 2). Subset analysis of clinical concordance per volume revealed that the SWI volumes had the highest clinical concordance with eight of 12 cases (case 7 had a non-diagnostic SWI volume). T2* and T2* N4 volumes had the second highest clinical concordance with seven of 13 cases.

\subsection{Concordance of surgical pathology to 7 T MRI findings}

Ten subjects underwent epilepsy surgery, of which seven had concordant histopathology with 7 T MRI findings and of these four cases had concordant HS (Fig. 3). In one out of the ten cases (case 2), while there was no structural cause for the TLE in the histopathology of the resected specimen, there was nevertheless complete concordance of 7 T MRI with the histopathology in this case. Three cases were discordant with 7 T MRI findings (cases 4, 7, 8). The results of each case are discussed in Table 2. Analysis of histopathological concordance per volume revealed that the SWI volumes had the highest histopathological concordance with seven of nine cases (case 7 had a non-diagnostic SWI volume). T2* N4 volumes had the second highest histopathological concordance with seven of ten cases.

\section{Discussion}

Previous studies have shown that 7 T MRI allows higher resolution depiction of the complex hippocampal anatomy [8,9,16-19]. Breyer et al. and Henry et al. $[9,19]$ applied imaging at $7 \mathrm{~T}$ in patients with clinical diagnosis of TLE and corresponding hippocampal abnormality on clinical $1.5 \mathrm{~T}$ or $3 \mathrm{~T}$ imaging. Breyer et al. had shown confirmation of HS (that was diagnosed at $1.5 \mathrm{~T}$ ) in all 6 of their patients on 7 T MRI [9]. However no pathological correlation was available for confirmation of the imaging findings in these two studies $[9,19]$. Coras et al. and Zucca et al. [20,21] have performed studies focusing on 7 T MR imaging of resected specimens in hippocampal sclerosis and focal cortical dysplasia which reveal the potential of high correlation between ex vivo imaging findings and histopathology of the resected specimens. Our study aimed to compare in vivo 7 T MRI imaging findings with histopathology.

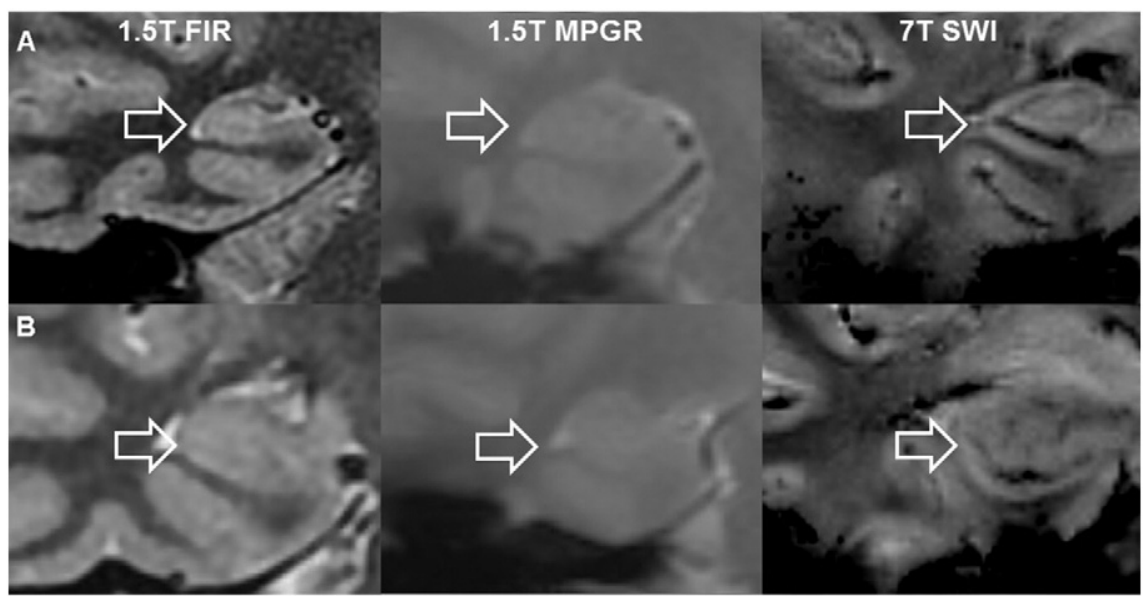

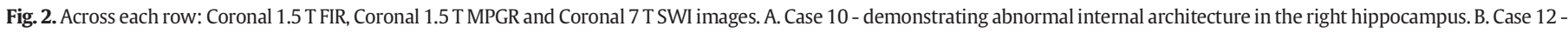

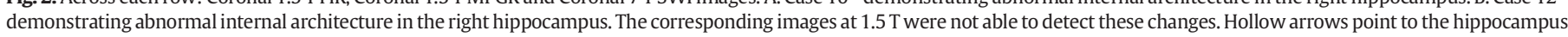
on all images. 

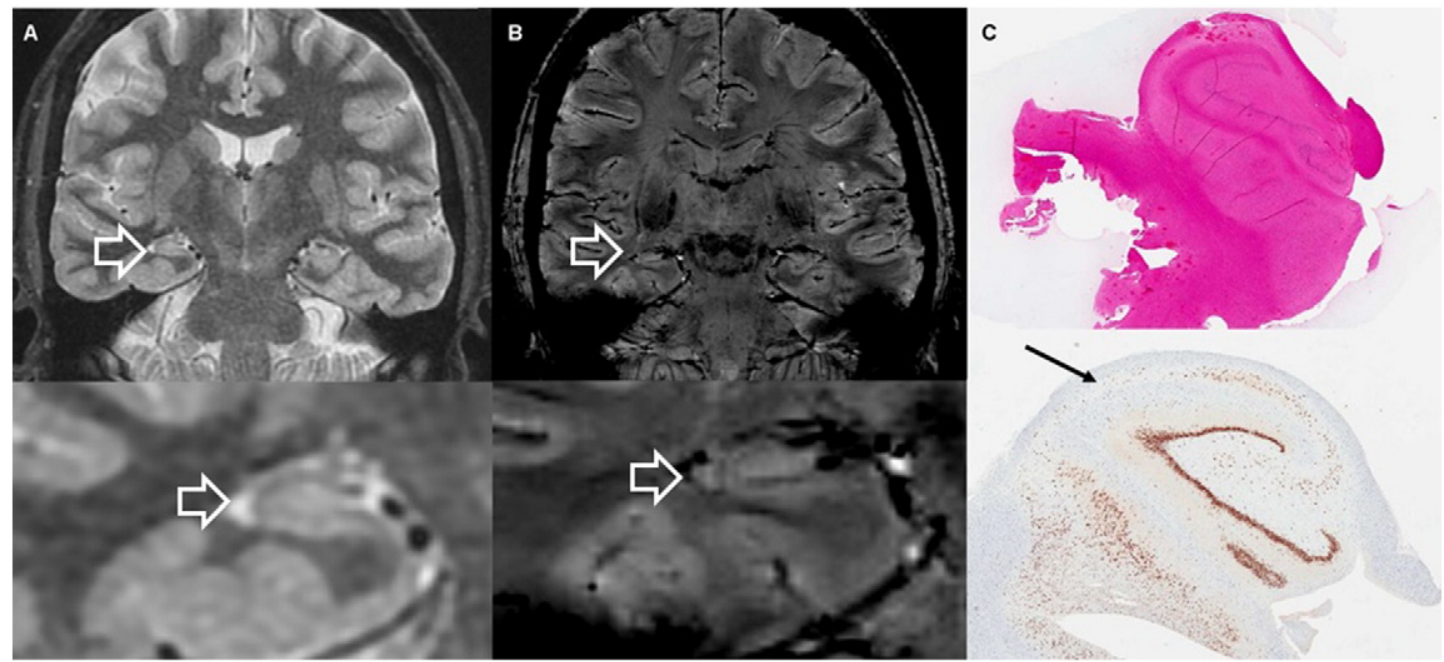

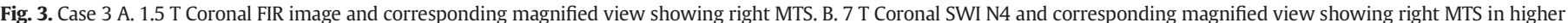

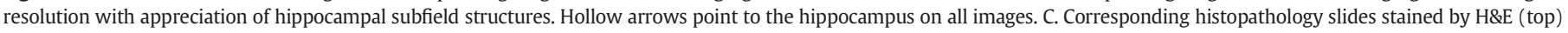
and NeuN (bottom) immunoperoxidase display chronic atrophy with marked neuronal loss (solid arrow).

The overall concordance of 7 T MRI findings and histopathology was high. There was complete concordance in patients with pathological proven HS suggesting that 7 T MRI can consistently detect this abnormality. This work can serve as a basis for validation of imaging findings at 7 T MRI. Discordant cases (cases 4, 7 and 8) had histopathology consisting of cortical dysplasia/gliosis, mild dentate fascia dispersion/ gliosis and focal cortical dysplasia type Ia/gliosis suggesting that $7 \mathrm{~T}$ MRI may still not have enough resolution to routinely detect these subtle findings. It is interesting to note that an abnormality was detected on $7 \mathrm{~T}$ MRI in these discordant cases but on the contralateral side (on both non-uniformity corrected and uniformity corrected sequences). These three patients have had good control of their seizures following resection which suggests that the 7 T MRI findings are false positives. Shading or susceptibility artifact (even after uniformity correction) may have played a role in this potential misinterpretation; this raises caution that imaging abnormalities should be interpreted in the clinical context. Additionally, as the $7 \mathrm{~T}$ MRI were interpreted while blinded to clinical data, directed search with clinical suspicion may have a role in increasing detection rates of subtle abnormalities. SWI and T2*-weighted nonuniformity-corrected volumes had the best concordance with histopathology; it is interesting to note that Breyer et al. also found that T2*weighted imaging at $7 \mathrm{~T}$ was valuable for anatomical delineation. [9].

Another point to note is that $1.5 \mathrm{~T}$ MRI performed similarly in both clinical and histopathological concordance to 7 T MRI. There was a slight apparent edge to 1.5 T MRI on clinical concordance, however this is likely a false positive coincidence as the suspected abnormality yielded normal histopathology (case 2). Overall, no clear advantage of 7 T MRI is noted for qualitative evaluation as compared to $1.5 \mathrm{~T}$. Subjectively, the image resolution at $7 \mathrm{~T}$ was felt to be of higher quality.

The limitations of this study include the small sample size and lack of an age and gender matched control group. More false positives may have been introduced since histopathological concordance was defined as any abnormal $7 \mathrm{~T}$ imaging finding with a corresponding ipsilateral abnormal histological finding, however this decision was made as the experience with 7 T MRI is relatively new and in this initial study a higher sensitivity approach was opted for to maximize the potential detection capabilities of 7 T MRI. Another potential limitation was that there may have been recall during blinded evaluation of the $1.5 \mathrm{~T}$ and $7 \mathrm{~T}$ MRI scans, however this was minimized as the $1.5 \mathrm{~T}$ scans were evaluated approximately 12 months after the 7 T MRI scans. Directed interpretation with clinical data may enhance the detection abilities at $7 \mathrm{~T}$. Additionally, a potential limitation is that a 3 T MRI comparison group was not available, as 3 T MRI was not available for clinical use at our institution during this study. Although it is important to note that 1.5 T MRI may still be more frequently used than 3 T MRI at many clinical centers.

\section{Conclusion}

$7 \mathrm{~T}$ MRI in patients presenting with epilepsy for pre-surgical workup may reveal findings not detected on lower field imaging. In patients undergoing epilepsy surgery, 7 T MRI findings had high correlation in our small series with the histopathology of the resected specimen. Pathologically proven hippocampal sclerosis was detected by 7 T MRI in all 4 patients which suggests it has the ability to reliably detect this finding. Discordance involving subtle findings such as focal cortical dysplasia and gliosis suggests that 7 T MRI may not reliably detect these findings and directed search with clinical information could be helpful. This experience also raises a cautionary flag to interpret $7 \mathrm{~T}$ MRI findings within the clinical context as false positive findings were suggested in the discordant cases; more experience with high field artifacts is needed to ensure correct interpretation. SWI and T2*-weighted volumes showed the highest clinical and histopathological concordance lending further credence that these sequences have superior anatomical depiction at high field strengths; it may be beneficial to consider the addition of these sequences for 7 T MRI in epilepsy workup. Qualitative hippocampal subfield analysis may be a potential application. Although a small study, no clear advantage of 7 T over 1.5 T MRI was observed for clinical and histopathological concordance. Future work with larger numbers and accompanying histopathology will be needed to determine the utility of 7 T MRI for epilepsy imaging and verify imaging findings.

\section{Authors' contributions}

Kwan: Protocol/project development, Data collection or management, Data analysis, Manuscript writing/editing

Salehi: Protocol/project development, Data collection or management, Data analysis, Manuscript writing/editing

Ohorodnyk: Protocol/project development, Data collection or management, Data analysis, Manuscript writing/editing

Lee: Protocol/project development, Data collection or management, Data analysis, Manuscript writing/editing

Burneo: Data analysis, Manuscript writing/editing

Mirsattari: Data analysis, Manuscript writing/editing

Steven: Data analysis, Manuscript writing/editing

Hammond: Data analysis, Manuscript writing/editing 
Table 2

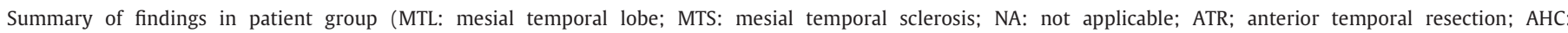
amygdalohippocampectomy).

\begin{tabular}{|c|c|c|c|c|c|c|c|c|c|c|}
\hline Case & Sex & $\begin{array}{l}\text { Age at } \\
\text { surgery }\end{array}$ & 1.5 T MR findings & 7 T MR findings & $\begin{array}{l}\text { Scalp EEG } \\
\text { localization }\end{array}$ & $\begin{array}{l}\text { Subdural } \\
\text { electrode } \\
\text { findings }\end{array}$ & $\begin{array}{l}\text { Surgery } \\
\text { performed }\end{array}$ & Pathology findings & $\begin{array}{l}\text { Follow } \\
\text { up } \\
\text { duration } \\
\text { (months) }\end{array}$ & $\begin{array}{l}\text { Engel } \\
\text { classification }\end{array}$ \\
\hline 1 & $\mathrm{~F}$ & 42 & $\begin{array}{l}\text { Right and left mesial } \\
\text { temporal lobe internal } \\
\text { architecture abnormality }\end{array}$ & $\begin{array}{l}\text { Right mesial temporal } \\
\text { lobe internal architecture } \\
\text { abnormality, right fornix } \\
\text { atrophy }\end{array}$ & Right temporal & Right MTL & $\begin{array}{l}\text { Right ATR, } \\
\text { AHC }\end{array}$ & $\begin{array}{l}\text { Gliosis (temporal } \\
\text { neocortex, hippocampus } \\
\text { and amygdala) }\end{array}$ & 17 & I \\
\hline 2 & M & 24 & $\begin{array}{l}\text { Right MTS, Left temporal } \\
\text { neocortical architecture } \\
\text { abnormality }\end{array}$ & Normal & Left temporal & Left MTL & $\begin{array}{l}\text { Left ATR, } \\
\text { AHC }\end{array}$ & Normal & 7 & I \\
\hline 3 & $\mathrm{~F}$ & 45 & Right MTS & Right MTS & Multifocal & Right MTL & $\begin{array}{l}\text { Right ATR, } \\
\text { AHC }\end{array}$ & $\begin{array}{l}\text { Gliosis (temporal lobe), } \\
\text { mesial temporal sclerosis }\end{array}$ & 39 & I \\
\hline 4 & M & 31 & $\begin{array}{l}\text { Right mesial temporal } \\
\text { internal architecture } \\
\text { abnormality }\end{array}$ & Left MTS & Bitemporal & NA & $\begin{array}{l}\text { Right ATR, } \\
\text { AHC }\end{array}$ & $\begin{array}{l}\text { Mild cortical dysplasia and } \\
\text { mild gliosis (temporal } \\
\text { neocortex), normal } \\
\text { hippocampus }\end{array}$ & 18 & I \\
\hline 5 & $\mathrm{~F}$ & 32 & Left MTS & Left MTS & Left temporal & NA & $\begin{array}{l}\text { Left ATR, } \\
\text { AHC }\end{array}$ & $\begin{array}{l}\text { Gliosis (temporal } \\
\text { neocortex), mesial } \\
\text { temporal sclerosis }\end{array}$ & 27 & I \\
\hline 6 & M & NA & Normal temporal lobes & Right MTS & $\begin{array}{l}\text { Left temporal, } \\
\text { parietal }\end{array}$ & NA & $\begin{array}{l}\text { No } \\
\text { resection }\end{array}$ & No resection & NA & NA \\
\hline 7 & M & 18 & $\begin{array}{l}\text { Left temporal neocortical } \\
\text { architecture abnormality }\end{array}$ & Left MTS & Right temporal & NA & $\begin{array}{l}\text { Right ATR, } \\
\text { AHC }\end{array}$ & $\begin{array}{l}\text { Gliosis (temporal } \\
\text { neocortex, hippocampus } \\
\text { and amygdala); mild } \\
\text { dentate fascia dispersion }\end{array}$ & 36 & I \\
\hline 8 & M & 23 & Normal temporal lobes & $\begin{array}{l}\text { Right mesial temporal } \\
\text { lobe internal architecture } \\
\text { abnormality }\end{array}$ & $\begin{array}{l}\text { Left } \\
\text { frontotemporal }\end{array}$ & Left MTL & $\begin{array}{l}\text { Left ATR, } \\
\text { AHC }\end{array}$ & $\begin{array}{l}\text { Gliosis (temporal } \\
\text { neocortex, hippocampus } \\
\text { and amygdala), focal } \\
\text { cortical dysplasia type Ia } \\
\text { (temporal neocortex) }\end{array}$ & 18 & I \\
\hline 9 & $\mathrm{~F}$ & 49 & $\begin{array}{l}\text { Right mesial temporal } \\
\text { lobe internal architecture } \\
\text { abnormality, Left } \\
\text { periventricular } \\
\text { heterotopia }\end{array}$ & $\begin{array}{l}\text { Right MTS, left mesial } \\
\text { temporal lobe size } \\
\text { abnormality }\end{array}$ & Multifocal & Right MTL & $\begin{array}{l}\text { Right ATR, } \\
\text { AHC }\end{array}$ & $\begin{array}{l}\text { Gliosis (temporal } \\
\text { neocortex), mesial } \\
\text { temporal sclerosis }\end{array}$ & 23 & II \\
\hline 10 & M & NA & $\begin{array}{l}\text { Left mesial temporal } \\
\text { internal architecture } \\
\text { abnormality, Left } \\
\text { periventricular } \\
\text { heterotopia }\end{array}$ & $\begin{array}{l}\text { Right mesial temporal } \\
\text { lobe internal architecture } \\
\text { abnormality }\end{array}$ & Multifocal & NA & $\begin{array}{l}\text { No } \\
\text { resection }\end{array}$ & No resection & NA & NA \\
\hline 11 & $\mathrm{~F}$ & 50 & $\begin{array}{l}\text { Left mesial temporal } \\
\text { internal architecture } \\
\text { abnormality, left } \\
\text { temporal neocortical } \\
\text { architecture abnormality }\end{array}$ & $\begin{array}{l}\text { Left mesial temporal lobe } \\
\text { size abnormality }\end{array}$ & Left temporal & NA & $\begin{array}{l}\text { Left ATR, } \\
\text { AHC }\end{array}$ & $\begin{array}{l}\text { Gliosis (temporal } \\
\text { neocortex, amygdala and } \\
\text { hippocampus) }\end{array}$ & 36 & IV \\
\hline 12 & $\mathrm{~F}$ & NA & $\begin{array}{l}\text { Left mesial temporal lobe } \\
\text { size abnormality }\end{array}$ & $\begin{array}{l}\text { Right mesial temporal } \\
\text { lobe internal architecture } \\
\text { abnormality, Left mesial } \\
\text { temporal lobe size } \\
\text { abnormality }\end{array}$ & $\begin{array}{l}\text { Bitemporal, } \\
\text { greater on } \\
\text { right }\end{array}$ & Bitemporal & $\begin{array}{l}\text { No } \\
\text { resection }\end{array}$ & No resection & NA & NA \\
\hline 13 & M & 20 & $\begin{array}{l}\text { Left MTS, left occipital } \\
\text { lobe encephalomalacia } \\
\text { (remote) }\end{array}$ & $\begin{array}{l}\text { Left MTS, left occipital } \\
\text { lobe encephalomalacia }\end{array}$ & $\begin{array}{l}\text { Left temporal, } \\
\text { occipital }\end{array}$ & Left MTL & $\begin{array}{l}\text { Left ATR, } \\
\text { AHC }\end{array}$ & $\begin{array}{l}\text { Gliosis (temporal } \\
\text { neocortex and amygdala), } \\
\text { mesial temporal sclerosis }\end{array}$ & 35 & I \\
\hline
\end{tabular}

Peters: Protocol/project development, Data analysis, Manuscript writing/editing

Khan: Protocol/project development, Data collection or management, Data analysis, Manuscript writing/editing

\section{Conflict of interest}

On behalf of all authors, the corresponding author states that there is no conflict of interest.

\section{References}

[1] L.J. Stovner, J.M. Hoff, S. Svalheim, et al., Neurological disorders in the global burden of disease 2010 study, Acta Neurol. Scand. Suppl. 198 (2014) 1-6.

[2] G.P. Winston, C. Micallef, B.E. Kendell, et al., The value of repeat neuroimaging for epilepsy at a tertiary referral centre: 16 years of experience, Epilepsy Res. 105 (2013) 349-355.
[3] S. Wiebe, W.T. Blume, J.P. Girvin, M. Eliasziw, A randomized, controlled trial of surgery for temporal-lobe epilepsy, NEJM 345 (5) (2001) 311-318.

[4] W.T. Blume, G.R. Ganapathy, D. Munoz, et al., Indices of respective surgery effectiveness for intractable nonlesional focal epilepsy, Epilepsia 45 (2004) 46-53.

[5] L. Jeha, I. Najm, W. Bingaman, et al., Predictors of outcome after temporal lobectomy for the treatment of intractable epilepsy, Neurology 66 (2006) 1938-1940.

[6] J.A. Sweet, A.M. Hdeib, A. Sloan, et al., Depths and grids in brain tumors: implantation strategies, techniques, and complications, Epilepsia 54 (Suppl. 9) (2013) 66-71.

[7] J.G. Burneo, D.A. Steven, R.S. McLachlan, A.G. Parrent, Morbidity associated with the use of intracranial electrodes for epilepsy surgery, Can. J. Neurol. Sci. 33 (2006) 223-227.

[8] B.P. Thomas, E.B. Welch, B.D. Niederhauser, et al., High-resolution 7 T MRI of the human hippocampus in vivo, J. Magn. Reson. Imaging 28 (2008) 1266-1272.

[9] T. Breyer, I. Wanke, S. Maderwald, et al., Imaging of patients with hippocampal sclerosis at 7 Tesla: initial results, Acad. Radiol. 17 (2010) 421-426.

[10] N.C. de Lanerolle, J.H. Kim, A. Williamson, et al., A retrospective analysis of hippocampal pathology in human temporal lobe epilepsy: evidence for distinctive patient subcategories, Epilepsia 44 (2003) 677-687.

[11] A. De Ciantis, A.J. Barkovich, M. Cosottini, et al., Ultra-high-field MR imaging in polymicrogyria and epilepsy, AJNR Am. J. Neuroradiol. 36 (2015) 309-316. 
[12] M.-L. Jones, S. Nikolova, J. Burneo, R. Bartha, 7 T MRS in medically refractory temporal lobe epilepsy patients with normal 1.5 T, American Epilepsy Society Annua Meeting Abstract 2014 (Abst. 3.277), December 5-9, 2014. Seattle, Washington, 2014.

[13] V. Prudent, A. Kumar, S. Liu, G. Wiggins, D. Malaspina, O. Gonen, Human hippocampal subfields in young adults at $7.0 \mathrm{~T}$ : feasibility of imaging, Radiology 254 (3) (2010) 900-906.

[14] M. Goubran, D. Rudko, B. Santyr, et al., In-vivo normative atlas of the hippocampa subfields using multi-echo susceptibility imaging at 7 Tesla, Hum. Brain Mapp. 35 (2014) 3588-3601.

[15] N.J. Tustison, B.B. Avant, P.A. Cook, et al., N4ITK: improved N3 bias correction, IEEE Trans. Med. Imaging 29 (2010) 1310-1320.

[16] J.M. Theysohn, O. Kraff, S. Maderwald, et al., The human hippocampus at $7 \mathrm{~T}-$ in vivo MRI, Hippocampus 19 (2009) 1-7.
[17] L.E.M. Wisse, L. Gerritsen, J.J.M. Zwanenburg, et al., Subfields of the hippocampal formation at 7 T MRI: in vivo volumetric assessment, NeuroImage 61 (2012) 1043-1049.

[18] P. Balchandani, T.P. Naidich, Ultra-high-field MR neuroimaging, AJNR Am. J. Neuroradiol. 36 (7) (2015) 1204-1215.

[19] T.R. Henry, M. Chupin, S. Lehéricy, et al., Hippocampal sclerosis in temporal lobe epilepsy: findings at 7 T, Radiology 261 (2011) 199-209.

[20] R. Coras, G. Milesi, I. Zucca, et al., 7 T MRI features in control human hippocampus and hippocampal sclerosis: an ex vivo study with histologic correlations, Epilepsia 55 (12) (2014) 2003-2016.

[21] I. Zucca, G. Milesi, V. Medici, et al., Type II FCD: ex vivo 7 Tesla MRI abnormalities and histopathological comparisons, Ann. Neurol. (2015) Epub ahead of print. 